\title{
Be stars in X-ray binary systems
}

\author{
M.J. Coe
}

Department of Physics and Astronomy, The University,

\begin{abstract}
.
This paper will review the status of our observations and understanding of Be stars in X-ray binary systems. In virtually all cases the binary partner to the Be star is a neutron star. The circumstellar disk provides the accretion fuel and hence stimulates the X-ray emission, whilst the neutron star provides a valuable probe of the environment around the $\mathrm{Be}$ star. The results coming from studies of such systems are helping in our understanding of the Be phenomenon.
\end{abstract}

\section{Introduction}

The objectives of this paper are :

- To explain what High Mass X-ray Binaries (HMXBs) are, and, in particular, Be/X-ray binary systems.

- To discuss the general observational characteristics of the group.

- To present examples of how work on such systems has furthered our knowledge of Be stars.

\section{General properties}

The $\mathrm{Be} / \mathrm{X}$-ray systems represent the largest sub-class of massive X-ray binaries. A survey of the literature reveals that of the 96 proposed massive X-ray binary pulsar systems, $67 \%$ of the identified systems fall within this classes of binary. The orbit of the Be or supergiant star and the compact object, presumably a neutron star, is generally wide and eccentric. X-ray outbursts are normally associated with the passage of the neutron star through the circumstellar disk. The optical star exhibits $\mathrm{H} \alpha$ line emission and continuum free-free emission (revealed as excess flux in the IR) from a disk of circumstellar gas.

The physics of accretion-powered pulsars has been reviewed previously (e.g. White, Nagase \& Parmar 1995, Nagase 1989, Bildsten et al. 1997). This paper will concentrate on the optical and IR observational properties of these systems and will address what such observations have contributed to our understanding of Be star behaviour.

$\mathrm{X}$-ray behavioural features of these systems include:

- regular periodic outbursts at periastron called Type I,

- giant outbursts at any phase probably arising from a dramatic expansion of the circumstellar disk described as Type II, 


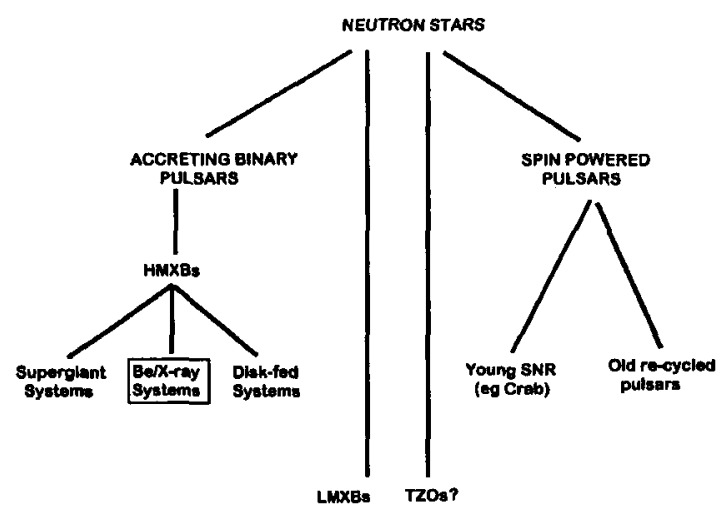

Figure 1. Classification of neutron star systems

- "missed" outbursts frequently related to low $\mathrm{H} \alpha$ emission levels (hence a small disk), or other unknown reasons (e.g. perhaps centrifugal inhibition of accretion (Stella, White \& Rosner 1986),

- shifting outburst phases (see below).

Progress towards a better understanding of the physics of these systems depends on a multi-wavelength programme of observations. From observations of the Be star in the optical and IR, the physical conditions under which the neutron star is accreting matter can be determined. In combination with hard $\mathrm{X}$-ray timing and flux observations, this yields a near complete picture of the accretion process. It is thus vital to identify the optical counterparts to these $\mathrm{X}$-ray systems in order to further our understanding.

Neutron stars are found in many astronomical configurations - see Figure 1 - and Be/X-ray binaries contain the second largest group of known neutron stars. The largest group comprises thousands of isolated rotation-powered pulsar systems.

Currently (September 1999) there are about 100 known or suspected HMXBs (see Figure 2). Surprisingly, nearly one third of all of these sytems lie in the Magellanic Clouds. This very large fraction, particularily noticeable in the Small Magellanic Cloud, will be discussed below in Section 7. Currently about one third of all the 100 systems have no known optical counterpart - frequently this is simply due to the inaccuracy of the X-ray observatory in locating the X-ray source.

There are two main sub-groups of HMXBS - the supergiant counterparts (normally of luminosity class I or II), and the Be/X-ray binary systems (normally luminosity class III or V). Both systems involve OB type stars and are commonly found in the galactic plane and the Magellanic Clouds. They differ, however, in accretion modes with the supergiant systems accreting from a radially outflowing stellar wind, and the Be/X-ray binaries accreting directly from the circumstellar disk (maybe with some limited Roche lobe overflow on rare occasions). As 


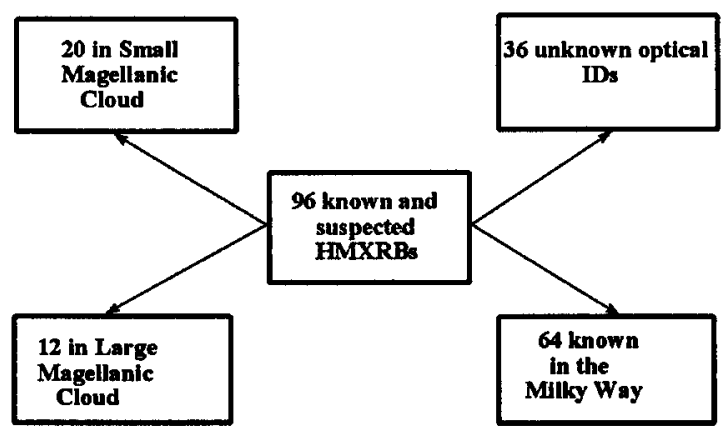

Figure 2. Distribution of HMXBs

a result the supergiants are persistent sources of $\mathrm{X}$-rays, whilst the $\mathrm{Be} / \mathrm{x}$-ray systems are very variable and frequently much brighter.

Recently Reig and Roche (1999) have suggested a third sub-group of systems; the X Persei like systems. The main characteristics of this proposed group are: long pulse periods (typically 1000s), persistent low X-ray luminosity $\left(\sim 10^{34}\right.$ $\mathrm{erg} / \mathrm{s}$ ) and low variability, and rare uncorrelated weak X-ray outbursts. There are currently 5 such objects in this new group.

A summary of the observational properties of $\mathrm{Be} / \mathrm{X}$-ray binaries is :

- they were first discovered in 1974-5 (eg A1118-616 and A0535+26),

- there are currently 38 optically identified systems,

- there are more 23 systems proposed based on X-ray characteristics,

- there are probably $100-1000 \mathrm{Be} / \mathrm{X}$-ray binaries in our galaxy (Bildsten et al. 1997), but maybe up to 10,000 (Rappaport \& van den Heuvel 1982, Meurs \& van den Heuvel 1989),

- binary periods lie in the range $16 \mathrm{~d}-400 \mathrm{~d}$,

- pulse periods lie in the range $0.07 \mathrm{~s}-1413 \mathrm{~s}$,

- the source names are primarily based upon their coordinates.

\section{Evolution}

The evolutionary history of Be stars in these $\mathrm{Be} / \mathrm{X}$-ray binary systems is somewhat different to that of their isolated colleagues. Figure 3 shows the widely accepted evolutionary path based upon conservative mass transfer that has been developed by van den Heuvel (1983) and Verbunt \& van den Heuvel (1995). The important consequences of the scenario is that wide binary orbits $(200-600 \mathrm{~d})$ are produced before the final supernova explosion. Hence, any small asymmetries in the subsequent SN explosion will then produce the frequently observed wide eccentric orbits.

Of particular interest is the narrow range of spectral class of the identified optical counterparts (see Negueruela 1998). In contrast to the sample of isolated $\mathrm{Be}$ stars to be found in the Bright Star Catalogue, there are no known Be/X-ray 


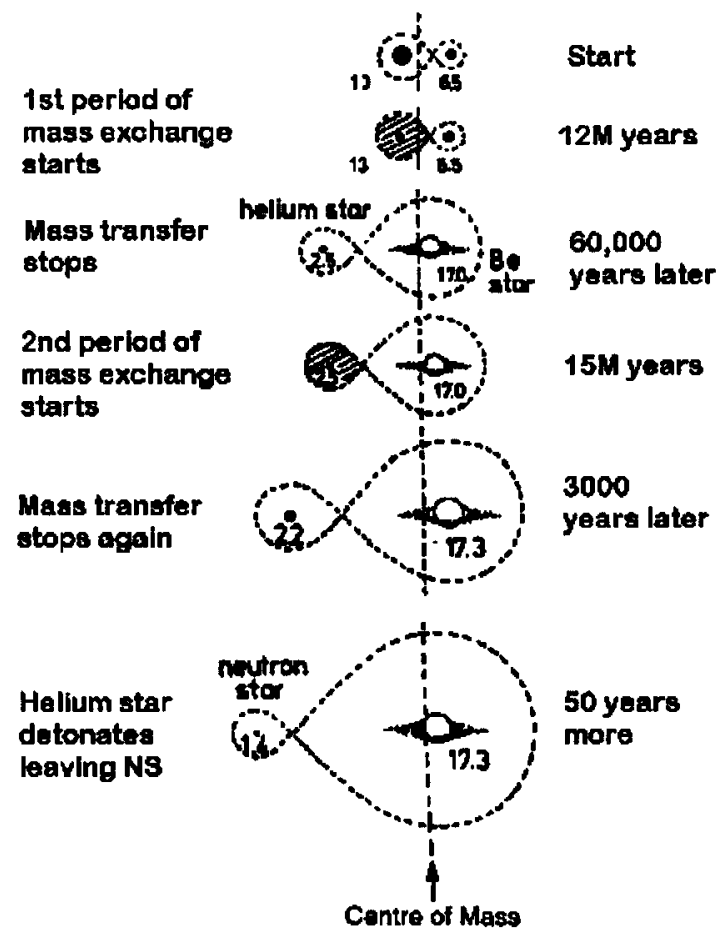

Figure 3. Evolution of a $\mathrm{Be} / \mathrm{X}$-ray binary system after van den Heuvel (1983) and others.

objects beyond spectral class B3 - see Figure 4. Most commonly the systems have counterparts in the B0-B2 group.

The explanation offered by Van Bever and Vanbeveren (1997) for this phenomenom is that the wide orbits produced by the evolutionary models are very vulnerable to disruption during the SN explosion. This will be particularily true for the less massive objects that would make up the later spectral classes. Hence the observed distribution is confirming the evolutionary models.

Some evolutionary models predict the existence of other Be star + compact object systems. In particular, the work of Raguzova and Lipunov (1999) discusses the emergence of $\mathrm{Be}+$ Black Hole systems and cites GRS 1915+105 as a possible example. Raguzova \& Lipunov (2000) also predict that " $46 \%$ of all Be stars formed in binary evolution should have White Dwarf companions". To date, however, only two B + WD systems are known (Vennes, Berghofer \& Christian 1997 and Burleigh \& Barstow 1999), and no Be + WD systems - but it is very hard to detect the WD in the presence of such bright companions. 


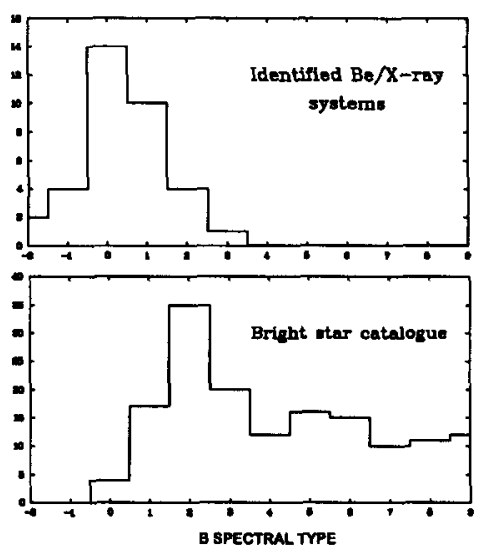

Figure 4. Comparison of the spectral classes of Be stars in Be/X-ray binaries (upper panel) with those in isolated systems (lower panel). Diagram adapted from Negueruela (1998) with permission.

\section{Periodicities}

One of the most exciting developments in the study of $\mathrm{Be} / \mathrm{X}$-ray binaries occurred when Corbet (1984) realised that a strong correlation exists between the spin period of the neutron star and the binary period of the system. Subsequently Corbet et al. (1999) developed this line of work and recently produced the more comprehensive diagram reproduced here as Figure 5. From this figure two things may be clearly seen: the intitial correlation for the $\mathrm{Be} / \mathrm{X}$-ray systems is strongly confirmed, and different classes of HMXB lie in distinctly different locations on the diagram. Hence not only does the diagram provide a valuable tool for estimating unknown binary periods in $\mathrm{Be} / \mathrm{X}$-ray systems, but if both periods are known then the class of object may be deduced.

The explanation for the striking relationship with the $\mathrm{Be} / \mathrm{X}$-ray binaries is to be found in the process of accretion on to the neutron star. For accretion to occur the Alfven radius must be less than the co-rotation radius of the accreting material. Otherwise the neutron star continues to spin down until this condition is met. However, the size of the Alfven radius depends upon the density of the surrounding medium and larger orbits mean lower stellar wind densities in the environment of the neutron star. Hence a relationship naturally develops between the orbital period and the equilibrium spin period of the neutron star.

\section{Example I: A1118-616}

The system A1118-616 provides an excellent example of a classic Be/X-ray binary system. It was discovered in 1991 by chance while the Ariel 5 satellite was observed the nearby source Cen X-3 (Eyles et al. 1975). Coe et al. (1994) show the time history of its X-ray flux over 2 months starting just before the 


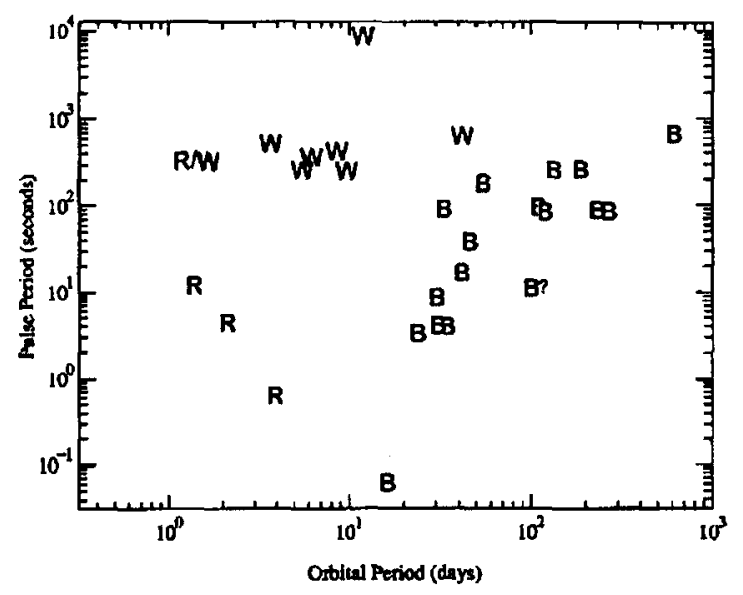

Figure 5. The relationship between spin period and orbital period for pulsating HMXBs. In the diagram $\mathrm{B}$ refers to $\mathrm{Be} / \mathrm{X}$-ray binaries, $\mathrm{R}$ to Roche-lobe overflow systems and $\mathrm{W}$ to wind-fed systems. Adapted from Corbet et al (1999) with permission.

initial outburst. During the outburst the flux of the source increased by much more than 1 order of magnitude and became the dominant hard X-ray source in the Centaurus region for 7-10 days. The X-ray source then disappeared from sight for 27 years before re-emerging in the same dramatic style in 1991.

The explanation for these sudden massive X-ray outbursts lies in the $\mathrm{H} \alpha$ observation carried out prior to, during, and after the 1991 outburst. Note, no similar observations exist for the 1974 outburst since the optical counterpart was not identified at that time. The history of the $\mathrm{H} \alpha$ observations over a 10 year period are shown in Figure 6. From this figure it is immediately apparent why the source went into outburst when it did - the $\mathrm{H} \alpha$ equivalent width had reached an exceptionally high value of more than $100 \AA$. Since the pulse period is $404 \mathrm{~s}$ the Corbet diagram suggests a binary period in the range $200-300 \mathrm{~d}$, therefore it is unlikely that the two recorded outbursts relate to binary motion (Type I outbursts). Far more likely, we are seeing two Type II outbursts, and the normal Type I outbursts are either very minor or don't occur at all. This could simply be due to the orbit not normally taking the neutron star through the circumstellar disk. However, during these abnormal levels of $\mathrm{H} \alpha$ activity, the disk probably expands to include the orbit of the neutron star, and hence accretion immediately begins. Note the strikingly rapid decline of the $\mathrm{H} \alpha$ immediately after the outburst suggesting that the whole period of activity was probably just due to one major mass ejection event from the Be star.

It is interesting to compare the size of the circumstellar disk seen in A1118 -616 with that observed in other systems. Figure 7 shows a plot of $\mathrm{H} \alpha \mathrm{EW}$ against the intrinsic infrared excess (also thought to arise from free-free and free-bound emission in the circumstellar disk). An obvious and unsurprising 


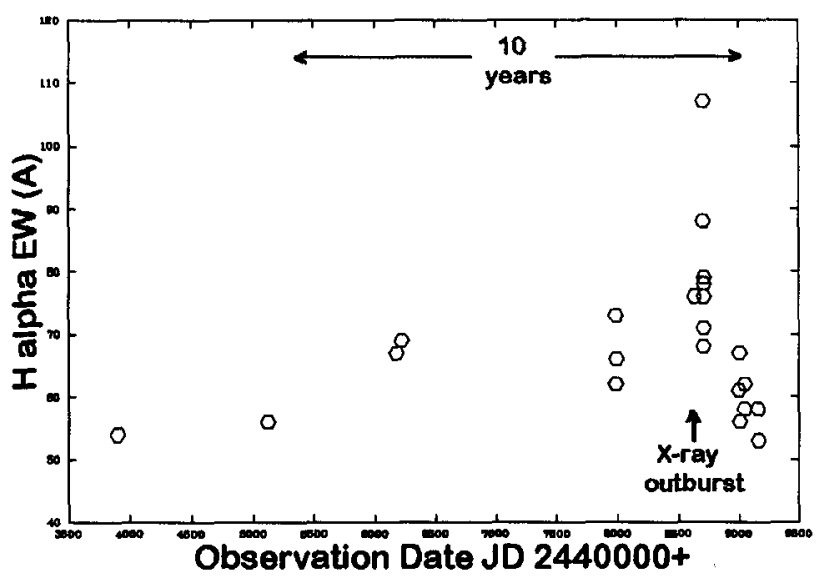

Figure 6. The history of the $\mathrm{H} \alpha$ emission from A1118-616 showing the large rise coincident with the X-ray outburst. From Coe et al. (1994)

correlation exists between these two parameters as is clear from the figure. The quiescent location of A1118-616 is shown on the diagram together with two other $\mathrm{Be} / \mathrm{X}$-ray systems. For comparison, data from a sample of isolated $\mathrm{Be}$ stars (Dachs and Wamsteker 1982) is also presented. It is interesting to note that even in quiescence, A1118-616 is at the extreme edge of the diagram, and in fact, its peak $\mathrm{H} \alpha \mathrm{EW}$ value of $\sim 110 \AA$ may be one of the very largest recorded values for any Be star.

\section{Example II: EXO2030+375}

The system EXO 2030+375 was discovered in the same manner as A1118-616 (a sudden outburst of activity from a previously unknown object), but by EXOSAT in 1985 (Parmar et al. 1989). The source was regularily monitored by the BATSE all sky instrument on the CGRO spacecraft (see Stollberg 1997 for an extensive discussion of this system). For long periods of time the source has shown classic Type I outbursts every periastron passage (binary period $=46 \mathrm{~d}$ ). Typical X-ray outbursts are shown in Figure 8 (Norton et al. 1994).

Then for about two years the source disappeared from the X-ray sky and re-merged in 1996. Detailed RXTE observations (Reig \& Coe 1998) revealed a significant shift in the binary phase of the outbursts of $\sim 0.15$. This phase has since remained unaltered, so possible precessional models of disks are ruled out. In addition, the $\mathrm{H} \alpha \mathrm{EW}$ had decreased from $-18 \AA$ to $-8 \AA$, thereby eliminating the possibility that the earlier outburst phase arose from to an expanded circumstellar disk which the neutron star encountered earlier in its orbit. It seems more likely that a fundamental change has occurred in the accretion mechanism 


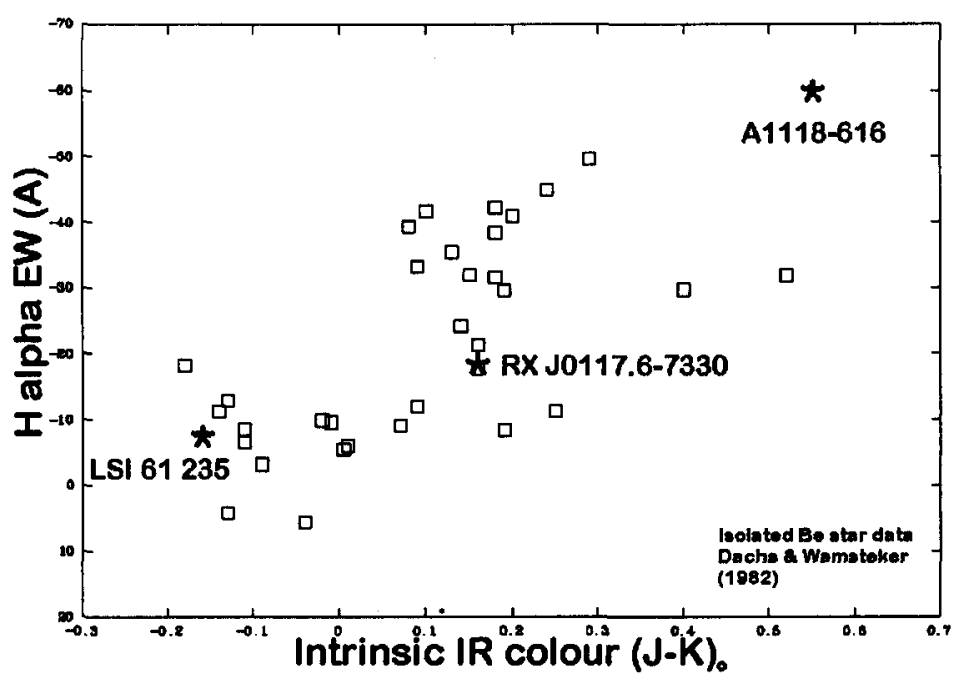

Figure 7. The relationship between the intrinsic IR colours and the $\mathrm{H} \alpha$ equivalent width. Three $\mathrm{Be} / \mathrm{X}$-ray binary systems are compared to a set of isolated Be stars. From Coe et al. (1994)
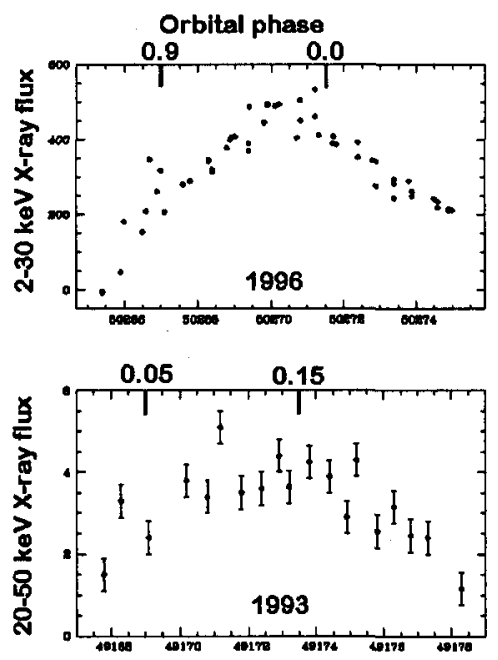

Figure 8. X-ray lightcurve of two outbursts from the system EXO $2030+375$. Note that the binary phase of the outburst has shifted significantly between 1993 and 1996. From Reig \& Coe (1998) with permission 


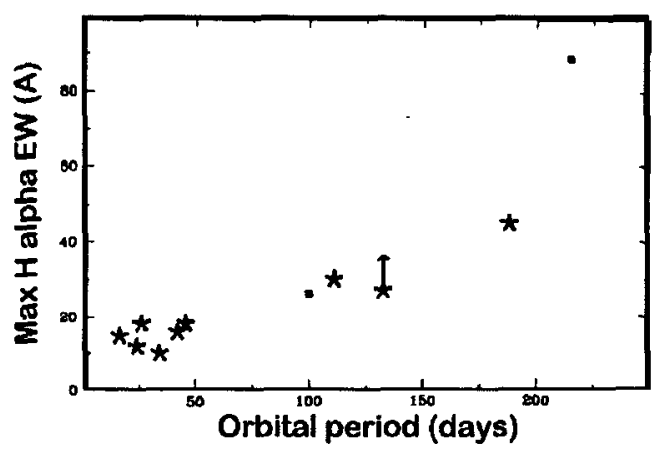

Figure 9. Comparison of the orbital period with the maximum $\mathrm{H} \alpha$ equivalent width for a variety of $\mathrm{Be} / \mathrm{X}$-ray systems. From Reig, Fabregat \& Coe (1997) with permission.

resulting from the presence of a much smaller disk. Probably, while the disk was large, the accretion was by Roche lobe overflow, but now it is simply by direct stellar wind accretion. This idea is supported by the very rapid pulse period changes seen prior to 1994 which had essentially ceased after 1996 - the more direct coupling of Roche lobe overflow would exert greater torques on the neutron star.

\section{Neutron star interactions with the circumstellar disk}

One of the particularily interesting questions related to studying $\mathrm{Be} / \mathrm{X}$-ray binaries is the question of the effect of the neutron star on the circumstellar disk. Initial work in this area by Norton et al. (1994) suggested that the dimensions of the circumstellar disk, compared with the amount of material undergoing accretion on to the neutron star, made it very unlikely that the presence of the neutron star would affect the growth of the circumstellar disk.

This view has now been altered by the longer term studies carried out by Reig, Fabregat \& Coe (1997). In Figure 9 the maximum observed $\mathrm{H} \alpha$ EW is plotted against the orbital period of several systems. Though the data are still rather sparse, there is a strong suggestion of a correlation. The conclusion the authors draw from this diagram is that the continually orbiting neutron star gradual erodes away the outer edges of the circumstellar disk and inhibits its growth.

Similarily, the same authors present a comparison of the maximum $\mathrm{H} \alpha$ EWs of Be stars in Be/X-ray binary systems with isolated Be stars. Again there is a strong suggestion that, on average, the disks are smaller in those systems with neutron star companions.

As yet the statistical sample sizes are small for these studies, but as more binary periods are determined these propositions can be checked. It is certainly 


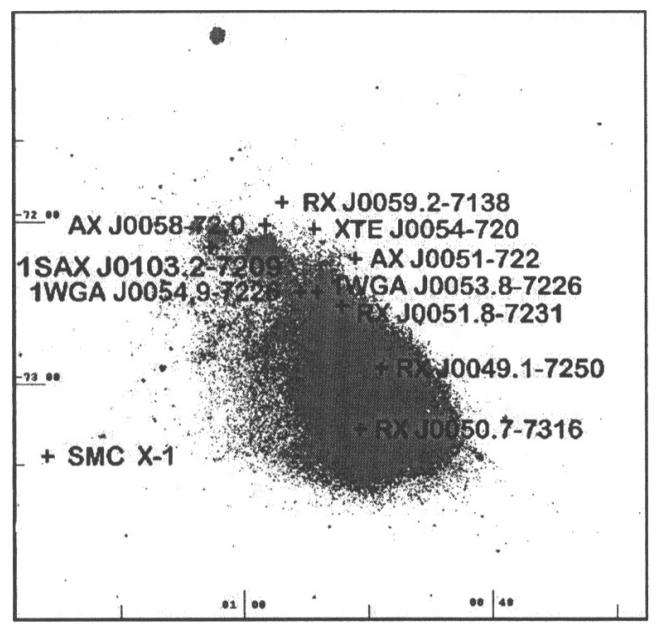

Figure 10. An optical image of the Small Magellanic Cloud showing the location of 11 out of the 20 known X-ray binary pulsar systems. From Coe and Orosz (2000)

important to understand how these two components in the binary system interact.

\section{Small Magellanic Cloud}

It has come as a great surprise to discover that there are a large number of $\mathrm{Be} / \mathrm{X}$ ray binaries in the Small Magellanic Cloud. Figure 10 shows the distribution of about half of these superimposed upon an optical image of the SMC.

It is possible to estimate the number of systems one would expect based upon the relative masses of our galaxy and the SMC. This ratio is $\sim 50$, so with 64 known or suspected systems in our galaxy we would only expect 1 or 2 systems in the SMC. However, Maeder, Grebel \& Mermilliod (1999) have shown that the fraction of Be stars to B stars is 0.39 in the SMC compared with 0.16 in our galaxy. So this raises the expected number of $\mathrm{Be} / \mathrm{X}$-ray systems to $\sim 3$ but we now know of 20 such systems!

The reason for the large number of Be stars almost certainly lies in the history of the Magellanic Clouds. Detailed H1 mapping by Stavely-Smith et al. (1997) and Putman et al. (1998) has shown a strong bridge of material between the Magellanic Clouds and between them and our own galaxy. Furthermore, Stavely-Smith et al. have demonstrated a the existence of a large number of supernova remnants of a similar age ( $\sim 5 \mathrm{Myr})$, strongly suggesting enhanced starbirth has taken place as a result of tidal interactions between these component systems. Consequently it seems very likely that the previous closest approach of the SMC to the LMC $\sim 100$ Myrs ago may have triggered the birth of many new massive stars which have given rise to the current population of 
HMXBs. In fact, other authors (eg Popov et al. 1998) claim that the presence of large numbers of HMXBs may be the best indication of starburst activity in a system.

Whatever the reason for the large number, the Small Magellanic Cloud now provides us with an excellent sample of $\mathrm{Be} / \mathrm{X}$-ray systems in a relatively compact and easily observed region of the sky.

\section{Conclusions}

Be stars in X-ray binary systems offer a whole new avenue for exploring the $\mathrm{Be}$ phenomenon. The evolutionary histories are different from the isolated systems, but their basic characteristics seem identical. The presence of a neutron star companion provides us with an extremely valuable probe of the circumstellar disk, and X-ray monitoring satellites provide one of the most rapid methods for identifying a Be star in outburst. Work in this field should continue to make substantial contributions to the much broader studies of these mysterious objects.

Acknowledgments. I am very grateful to my many collaborators over the years who have made major contributions to the topics discussed in this paper. In particular, I wish to thank Dave Buckley (SAAO), Simon Clark (Sussex), Juan Fabregat (Valencia), Ignacio Negueruela (Rome), Andy Norton (OU), Pablo Reig (Crete), Paul Roche (Leicester) and Iain Steele (Liverpool).

I also wish to thank the organisers of IAU Colloquium 175 for giving me an opportunity to discuss the work in this field.

\section{References}

Bildsten, L., Chakrabarty, D., Chiu, J., Finger, M.H., Koh, D., Nelson, R.W., Prince, T.A., Rubin, B.C., Scott, D.M., Stollberg, M., Vaughan, B.A., Wilson, C.A. \& Wilson, R.B. 1997, ApJS, 113, 367

Burleigh, M.R. \& Barstow, M.A. 1999, A \& A 341, 795

Coe, M.J., Roche, P., Everall, C., Fishman, G.J., Hagedon, K.S., Finger, M., Wilson, R.B., Buckley, D.A.H., Shrader, C., Fabregat, J., Polcaro, V.F., Giovannelli, F. \& Villada, M. 1994, A \& A, 289, 784.

Coe, M.J. \& Orosz, J.A. 2000, MNRAS, 311, 169

Corbet, R.H.D. 1984, A \& A, 141, 91

Corbet, R.H.D., Marshall, F.E., Peele, A.G. \& Takeshima T. 1999 ApJ, 517, 956

Dachs, J. \& Wamsteker, W. 1982, A \& A, 107, 240

Eyles, C.J., Skinner, G.K., Wilmore, A.P. \& Rosenberg, F.D. 1975, Nature, 254, 577

Maeder, A., Grebel, E.K. \& Mermilliod, J.C. 1999, A \& A, 346, 459

Meurs, E.J.A. \& van den Heuvel E.P.J. 1989, A \& A, 226, 88

Nagase, F. 1989 PASJ, 41, 1 
Negueruela, I. 1998 A \& A, 338, 505

Norton, A.J., Chakrabarty, D., Coe, M.J., Everall, C., Finger, M.H., Prince, T.A., Roche, P., Stollberg, M.T. \& Wilson, R.B. 1994, MNRAS, 271, 981

Parmar, A.N., White, N.E., Stella L., Izzo C., Ferri, P. 1989, ApJ, 338, 359

Popov, S.B., Lipunov, V.M., Prokhorov, M.E. \& Postnov, K.A. 1998, Astron Reports, 42,29

Putman, M.E. and 25 other authors 1998, Nature, 394, 752

Raguzova, N.V. and Lipunov, V.M. 1999, A \& A, 349, 505

Raguzova, N.V. and Lipunov, V.M. 2000, this conference, p.693

Rappaort S. \& van den Heuvel E.P.J. 1982, in Be Stars, Proceedings of the Symposium, (Dordrecht: Reidel), 327

Reig, P., Fabregat, J. \& Coe, M.J. 1997, A \& A, 322, 193

Reig, P. \& Coe, M.J. 1998, MNRAS, 294, 118

Reig, P. \& Roche, P. 1999, MNRAS, 306, 100

Stavely-Smith, L., Sault, R.J., Hatzidimitriou, D., Kesteven, M.J. \& McConnell, D. 1997, MNRAS, 289, 225

Stella, L., White, N.E. \& Rosner, R. 1986, ApJ, 308, 669

Stollberg, M.T. 1997, PhD Thesis, University of Alabama in Huntsville

van den Heuvel, E.P.J. 1983, in Accretion driven stellar X-ray sources, eds Lewin, W.H.G. \& van den Heuvel, E.P.J., (Cambridge University Press)

Van Bever, J. \& Vanbeveren, D. 1997, A \& A, 322, 116

Vennes S., Berghofer, T.W. \& Christian, D.J. 1997, ApJ, 491, L85

Verbunt, F. \& van den Heuvel, E.P.J. 1995, in X-ray Binaries, eds. Lewin, W.H.G., Van Paradijs J. \& van den Heuvel, E.P.J., (Cambridge University Press), p. 457

White, N.E., Nagase, F. \& Parmar, A.N. 1995, in X-ray Binaries, eds. Lewin, W.H.G., Van Paradijs J. \& van den Heuvel, E.P.J., (Cambridge University Press), p. 1

\section{Discussion}

N. Raguzova: I would like to make some comments about Be stars with white dwarfs. All white dwarfs in such systems must have very high effective temperatures and we can detect such objects in the extreme ultraviolet range.

M. Coe: Yes, that is correct. The two known B/WD systems have been found with EUVE - though it is proving much more difficult than one might think. Some people believe that the numbers of such systems may not be as large as you suggest.

W. Hummel: Are the detected Be/X-ray binaries in the SMC field stars, or members of open clusters?

M. Coe: There is no evidence so far from the identified systems that they are mainly found in clusters. 